\title{
Cegueira cortical: relato de dois casos clínicos
}

\author{
Cortical blindness: report of twocases
}

\author{
Raquel de Lima e Silva ${ }^{1}$ \\ Carlos Gustavo Leite Vieira ${ }^{1}$ \\ Marcelo Ramos ${ }^{2}$ \\ Rogério Soares Marques² \\ Renato Cruz Laender ${ }^{3}$
}

\section{RESUMO}

O objetivo deste trabalho é relatar dois casos de cegueira cortical atendidos no serviço de urgência da Clínica de Olhos da Santa Casa de Belo Horizonte. A cegueira cortical é uma condição clínica rara e bilateral, de causa isquêmica, caracterizada por lesão no córtex cerebral. Nos presentes casos, o diagnóstico foi feito pela sintomatologia clínica, pelos achados oftalmológicos e pelas alterações obtidas por imagem. Em ambos houve evolução rápida da doença, com perda visual súbita importante. O acompanhamento oftalmológico não revelou melhora significativa da acuidade visual final. A baixa acuidade visual é um sinal importante em oftalmologia e deve ser avaliada com cautela e atenção, visto que pode ser causada pela cegueira cortical, condição rara, grave e ainda pouco estudada no nosso meio.

Descritores: Cegueira cortical/diagnóstico; Tomografia computadorizada por raios $\mathrm{x}$; Transtornos da visão; Relato de caso

\section{INTRODUÇ̃̃̃O}

A cegueira cortical é uma condição clínica rara caracterizada por baixa acuidade visual, causada por lesão retrogeniculada bilateral ${ }^{(1)}$. Essa doença é provocada por lesão das radiações ópticas, direita e esquerda, por lesão da radiação óptica de um lado e lesão da área estriada do outro lado ou ainda, por lesão de ambas as áreas estriadas. As lesões dos hemisférios unilaterais não interferem na acuidade visual, entretanto o envolvimento bilateral das vias retrogeniculadas ou córtex occipital pode produzir defeitos no campo visual que diminuem a acuidade visual em qualquer grau. $\mathrm{O}$ acidente vascular cerebral é a principal causa da doença ${ }^{(2-3)}$. Os principais fatores de risco: hipertensão arterial sistêmica, diabetes mellitus, hipercolesterolemia, cardiopatias, doenças ateromatosas dos vasos do pescoço, tabagismo e estresse. Huber \& Grob em 1998 relataram um caso de cegueira cortical bilateral após cirurgia da espinha lombar ${ }^{(4)}$. No sexo feminino ainda pode ser acrescentado como fatores de risco o uso de anticoncepcionais orais e a reposição hormonal no climatério ${ }^{(5)}$. A doença ainda pode estar associada a várias patologias sistêmicas, como no quadro $1^{(1)}$.

O diagnóstico pode ser estabelecido pela baixa súbita da acuidade visual, por alterações do campo visual e pela presença de áreas de infarto, principalmente no território da artéria cerebral posterior, confirmado por tomografia computadorizada ${ }^{(6)}$. Uma das raras complicações da doença é a Síndrome de Anton que envolve centros associados à visão, condição na qual o paciente nega a cegueira ${ }^{(1,5)}$. A cegueira cortical pode facilmente ser confundida com cegueira factícia, visto que as duas síndromes apresentam baixa acuidade visual, reações pupilares normais, meios claros e fundoscopia normal ${ }^{(2)}$.

Os casos que se seguem, atendidos na Clínica de Olhos da Santa Casa 


\begin{tabular}{|ll|}
\hline \multicolumn{2}{|c|}{ Quadro 1. Causas associadas à etiologia da cegueira cortical } \\
Choque & Meningite; encefalite \\
Pós angiografia & Esclerose múltipla \\
Pós "bypass" cardiopulmonar & Panencefalite esclerosante múltipla \\
Hipotensão & Síndrome de Creutzfeldt-Jakob \\
Pós ventriculografia & Leucodistrofia adrenal \\
Hipoglicemia & Edema e tumor cerebral \\
Mercúrio; etanol & Malformações congênitas cerebrais \\
Cis platinum & Encefalopatia mitocondrial \\
Porfiria intermitente & \\
*Fonte: Duane`s Clinical Ophthalmology \\
\hline
\end{tabular}

de Belo Horizonte, apresentaram quadro clínico de cegueira cortical, cujo diagnóstico foi estabelecido de acordo com os critérios referidos acima, isto é, clínicos e por imagem.

\section{RELATO DE CASOS}

\section{Caso 1}

Em 17/9/98, W.M., masculino, 51 anos, diabético e hipertenso apresentava perda de visão bilateral há 5 horas. O exame inicial apresentava ausência de percepção luminosa bilateral, nenhuma alteração à biomicroscopia, pressão intra-ocular normal e reflexos pupilares normais. A fundoscopia apresentou atenuação arteriolar, presença de hemorragias discretas em chama de vela e poucos exsudatos algodonosos em pólo posterior - retinopatia esclero-hipertensiva. A tomografia computadorizada do crânio revelou área de infarto isquêmico cerebral em ambos hemisférios (Figura 1). A avaliação cardiológica através do exame clínico e do ecocardiograma revelou miocardiopatia segmentar do ventrículo esquerdo, déficit sistólico global importante, insuficiência mitral e tricúspide discreta e grande trombo apical no ventrículo esquerdo. O paciente foi acompanhado durante 8 meses e não apresentou melhora da acuidade visual.

\section{Caso 2}

Em 09/01/99, V.S.S., masculino, 65 anos, queixava perda súbita da visão bilateral há quatro dias. Relatava visão prévia normal e negava episódio similar anterior. Tabagista há 20 anos e hipertenso com mal controle clínico, negava uso de medicação e negava também diabetes mellitus ou outras patologias. Ao exame clínico a sua visão era de percepção luminosa no olho esquerdo e ausência de percepção luminosa no olho direito. Em ambos os olhos a biomicroscopia estava
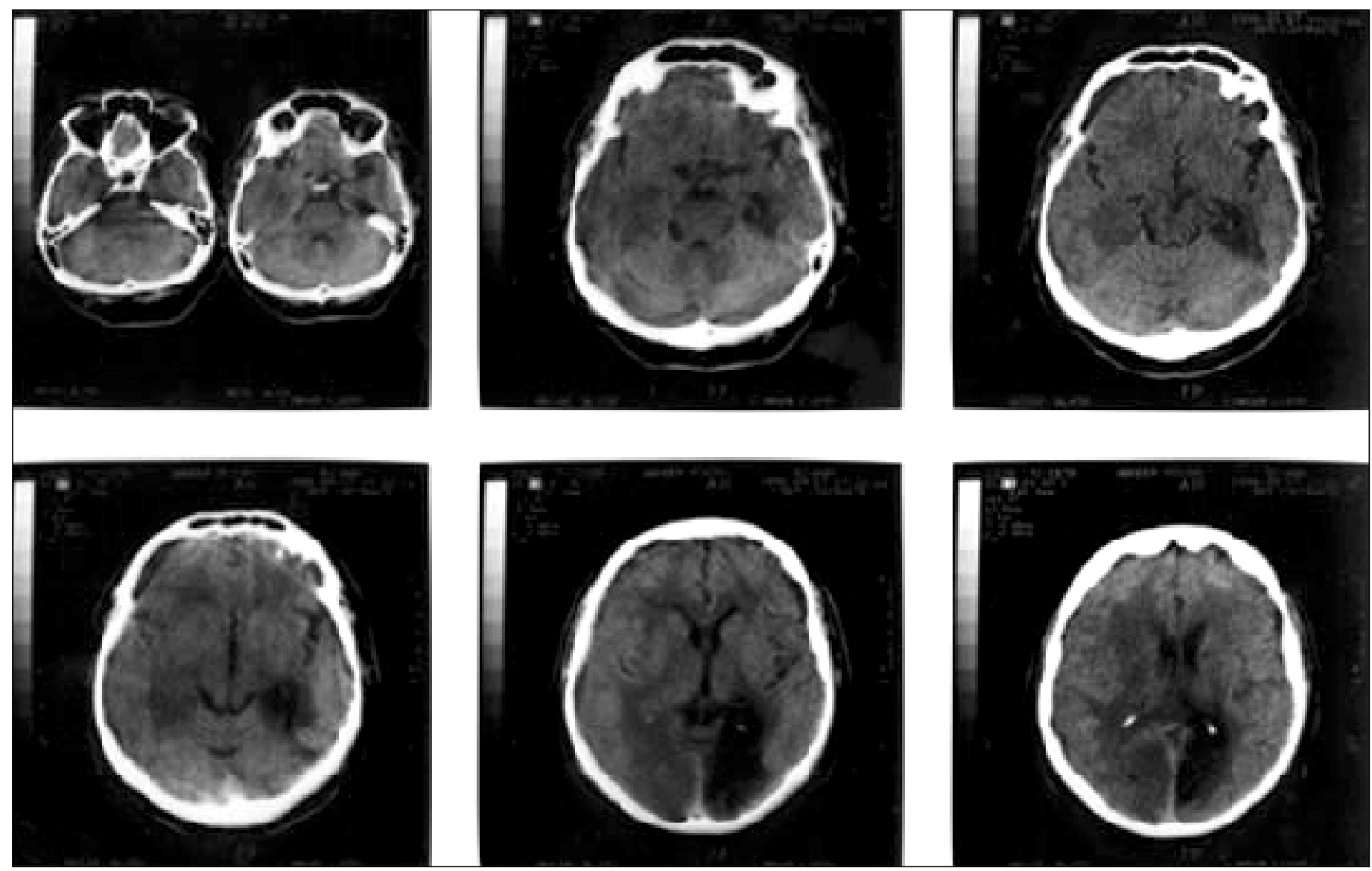

Figura 1 - Infarto isquêmico cerebral, intenso à esquerda 
normal, a pressão intra-ocular encontrava-se em seu limite superior $(20 \mathrm{mmHg})$ e os reflexos pupilares estavam ligeiramente diminuídos. À fundoscopia notava-se oclusão venosa da arcada temporal no olho direito e ausência de alterações no olho esquerdo. A tomografia computadorizada (11/01/99) revelou área hipodensa occipital bilateral, infartos seqüelares occipitais bilaterais e ateromatose bicarotideana. A arteriografia apresentou (Figuras 2 A e B) ateroma na carótida interna direita, aterosclerose difusa em todo encéfalo (bilateralmente), obstruções arteriais significativas e placas ateromatosas em todo trajeto basilar. O paciente foi acompanhado por um ano e apresentou melhora da acuidade visual para percepção luminosa no olho direito e manteve a percepção luminosa no olho esquerdo.

\section{COMENTÁRIOS}

No caso 1, a presença de um êmbolo de origem cardíaca pode ter tido importância no aparecimento do evento isquêmico cerebral. A embolização é uma causa freqüente de obstrução da circulação retiniana e êmbolos cardíacos ou carotídeos são os mais comuns ${ }^{(7-8)}$. A oftalmoscopia binocular indireta não mostrou sinais que justificassem a ausência de percepção luminosa bilateral. O campo visual neste caso foi inviável pela ausência de percepção de luz. A tomografia computadorizada de crânio (Figura 1) auxiliou o diagnóstico ao revelar área de infarto isquêmico cerebral em ambos os hemisférios. A alteração fundoscópica no segundo caso (oclusão venosa da arcada temporal em olho direito) poderia dificultar o diagnóstico, entretanto foi detectada somente no olho direito enquanto que a perda visual foi bilateral. Tais achados fundoscópicos poderiam, provavelmente, ter surgido antes da cegueira cortical ou concomitantes, uma vez que tanto a oclusão venosa da retina quanto a cegueira cortical bilateral possuem como principais fatores de risco hipertensão arterial sistêmica e doenças cardiovasculares $^{(7)}$. A tomografia computadorizada de crânio revelou infartos seqüelares occipitais bilaterais e ateromatose bicarotideana. Neste caso também havia alterações vasculares e isquêmicas à arteriografia (Figuras 2A e B). Em ambos os casos os pacientes apresentaram diminuição da acuidade visual, reflexos pupilares praticamente normais e ausência de alterações na oftalmoscopia binocular indireta. Tal quadro e os achados tomográficos caracterizaram o diagnóstico $^{(9)}$. O acompanhamento subseqüente dos pacientes foi realizado simultaneamente por neurologista, cardiologista e oftalmologista. O primeiro caso foi acompanhado pelo oftalmologista por 8 meses e, atualmente, faz controle ambulatorial. Não ocorreu melhora da acuidade visual em nenhum dos olhos. O segundo paciente foi submetido, no Departamento de Retina, a fotocoagulação da oclusão de ramo venoso em olho direito e encontra-se em controle ambulatorial. Apresentou melhora da acuidade visual para percepção luminosa no olho direito e manteve percepção luminosa no olho esquerdo, depois de 1 ano de seguimento. De acordo com a literatura médica, o prognóstico da cegueira cortical é reservado, mas em alguns casos pode haver o retorno gradual da visão até percepção luminosa e de movimentos de mãos ${ }^{\left({ }^{9}\right)}$. Normalmente o prognóstico depende de causa, gravidade, duração da doença e melhoria inicial rápida do quadro ${ }^{(5)}$. Aldrich e colaboradores em 1987 observaram que em 15 pacientes com cegueira cortical o melhor prognóstico foi observado naqueles com menos de 40 anos, sem fatores de risco, como hipertensão arterial sistêmica e diabetes mellitus, e sem alterações cognitivas, de linguagem ou de memória ${ }^{(9)}$. Nos presentes casos, a acuidade visual não melhorou, a não ser no segundo caso, no qual ocorreu leve melhora da percepção luminosa em olho direito. Levando em conta que ambos tinham mais de 40 anos de idade, hipertensão arterial e um deles também diabetes mellitus, pode-se dizer que a evolução e o quadro clínico de

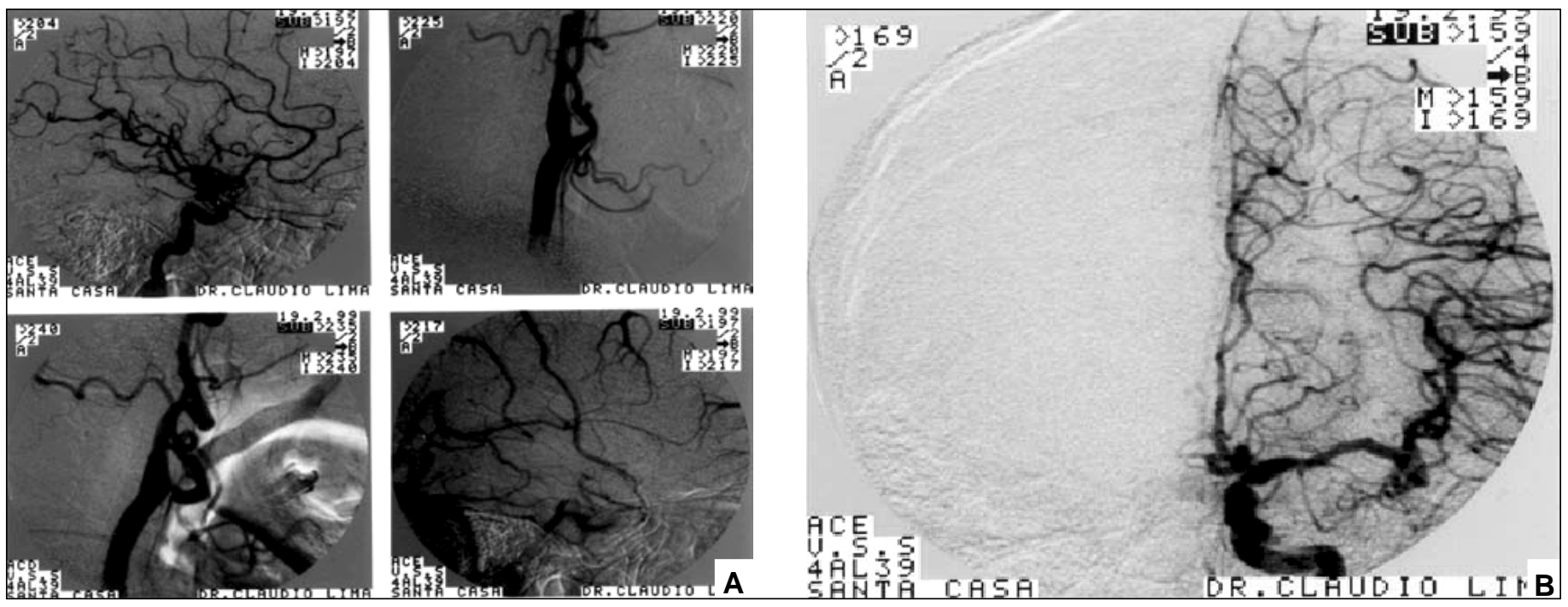

Figura 2 - A: Obstruções arteriais e ateromatose da artéria carótida; B: Alargamento e tortuosidade vascular 
ambos estão de acordo com os dados observados na literatu$\mathrm{ra}^{(10)}$. Cuellar \& Sanchez também relataram uma melhora da acuidade visual em poucos dias de evolução, em pacientes jovens com cegueira cortical pós-operatória ${ }^{(11)}$.

A baixa acuidade visual é um sinal importante em oftalmologia e deve ser avaliada com cautela e atenção, visto que pode ser causada pela cegueira cortical, condição rara, grave e ainda pouco estudada no nosso meio.

\section{ABSTRACT}

The aim of this work is to report two cases of cortical blindness, evaluated at an ophthalmologic service (Clínica de Olhos da Santa Casa de Belo Horizonte). Cortical blindness is a rare, usually ischemic, bilateral retrogeniculate lesion. The two diagnoses were based on patient complaints, ophthalmologic data and image signs. Both patients suddenly lost their visual function. None presented a significant improvement in their final vision acuity during the evolution. Subnormal visual acuity is an important ophthalmologic sign that must be seen with concern and attention since it may be caused by cortical blindness, a rare and serious condition not yet well studied in our Country.
Keywords: Cortical blindess/diagnosis; Tomography, x-ray computed; Vision disorders; Case report

\section{REFERÊNCIAS}

1. Tasman W, Jaeger EA editors. Duane's clinical ophthalmology. Philadelphia: JB Lippincott; 1991. p. 15-6.

2. Duke-Elder S, Scott GI. System of Ophthalmology. Neuro-Ophthalmology 1996; $12: 432$

3. Milandre L, Brosset C, Botti G, Khalil R. A study of 82 cerebral infarctions in the area of posterior cerebral arteries. Rev Neurol (Paris) 1994;150:133-41.

4. Huber JF, Grob D. Bilateral cortical blindness after lumbar spine surgery. A case report. Spine 1998;23:1807-9.

5. Argenta PA, Morgan MA. Cortical blindness and Anton syndrome in a patient with obstetric hemorrhage. Obstet Gynecol 1998;91(5Pt 2):810-2

6. Chatterjee A, Southwood H. Cortical Blindness and Visual Imagery. Neurology 1995;45:2189-95.

7. Kanski JJ. Clinical ophthalmology: a systemic approach. $3^{\text {rd }}$. ed. London: Butterworths; 1991. p. 357-70.

8. Futrell N, Millikan C, Watson BD, Dietrich WD, Ginsberg MD. Embolic stroke from a carotid arterial source in the rat: pathology and clinical implications. Neurology 1989;8:1050-6.

9. Aldrich MS, Alessi AG, Beck RW, Gilman S. Cortical blindness: etiology, diagnosis, and prognosis. Ann Neurol 1987;21:149-58.

10. Miller NR. Cerebrovascular disease. In: Walsh FB, Hoyt WE, Miller NR, eds. Walsh and Hoyt's Clinical Neuro-Ophthalmology. $4^{\text {th }}$ ed. Baltimore: Williams \& Wilkins, 1994:2362-7.

11. Cuellar E, Diaz-Sanchez F, Hinojosa A, Gonzales-Jimenez JM, RodriguezRodriguez JM, Munoz-Guardia JL. Ceguera cortical postoperatoria tras reseccion de teratocarcinoma retroperitoneal. Rev Esp Anestesiol Reanim 1995;42:178-81.

\title{
I Simpósio do Instituto de Oftalmologia Professor Ivo Corrêa-Meyer
}

\author{
26 e 27 de Outubro de 2001 \\ Porto Alegre - RS
}

INFORMAÇÕES: Tel: (00xx51)346-3636

e-mail: iicm@ig.com.br 